\title{
Application of Fuzzy Logic in Design of an Aesthetics-Based Interactive Architectural Space
}

\author{
Asma $\operatorname{Naz}^{1}$ and Mihai Nadin ${ }^{2 *}$ \\ ${ }^{1}$ Ph.D., ORCID 0000-0002-0584-1314, Researcher, Institute for Research in Anticipatory Systems, The University of \\ Texas at Dallas, Richardson, TX, USA \\ ${ }^{2}$ Ph.D, ORCID 0000-0001-9712-8921, Institute for Research in Anticipatory Systems, Ashbel Smith University Professor, \\ The University of Texas at Dallas, Richardson \\ (*Corresponding author) Email id:*nadin@utdallas.edu,'Iasma.naz@utdallas.edu
}

\begin{abstract}
This paper presents a successful application of fuzzy logic in the design of an interactive control system for an architectural living space. Fuzzy logic is applied in order to process subjective perceptual data based upon aesthetic considerations. In interactively modifiable living space, occupants can generate various sensony-perceptive spatial qualities with affective dimensions in real time. This is accomplished by modifying one or more design parameters of color, brightness, texture, and material in order to meet the emotional, psychological, proprioceptive and aesthetic needs associated with daily living. The human-space interaction is perception-based. It is structured around a set of aesthetic guidelines formulated from correlations that measure the extent to which each design parameter impacts user perception. Fuzzy logic system implements the aesthetic guidelines as fuzzy control rules in the analysis. It allows for processing non-numerical, linguistic data in order to modify appropriate design parameters and produce spatial outputs that are meaningful and effective for the occupants.
\end{abstract}

Keywords: Aesthetics, Affective space, Fuzzy logic system, Perception, Interaction, Architectural design

\section{INTRODUCTION}

This paper elaborates on the design process of a control system that regulates human-space interaction for an interactively modifiable living space. Fuzzy logic was selected as an appropriate mathematical model for designing interaction for this space. It is a soft computational method that is applied for computing, interpreting, and processing of subjective input and output data based upon a set of aesthetic principles. Qualitative data processing for architectural spaces require interpretation of linguistic data pertaining to perceptions and opinions regarding spatial qualities. The suggestion to work with fuzzy logic for this problem comes from ${ }^{[1]}$ and ${ }^{[2]}$.

The interactively modifiable living space introduced in this paper is a concept of a real-world dwelling proposed as a living solution for neo-nomads, that is, the mobile tech professionals popularly known as Silicon Valley workers ${ }^{[3]}$. It is a single room with six enclosing surfaces-four walls, a ceiling and a floor-with modifiable design parameters of color, brightness, texture, and material. Interaction with space enables the occupants the capability to articulate space, i.e., modify the design parameters as variables of visual space perception in order to change the feel and appearance of the space as a whole. Through spatial articulation-a space creation technique of traditional architecture-the design aims to accommodate the occupant's daily lifestyle. This involves accommodation of their emotional, psychological, proprioceptive and aesthetic needs. 
The human-space interaction of this design is based upon the premise that sensory design parameters of color, brightness, material, and texture have an impact on an occupant's emotional responses to the living space. The interrelationship of architectural elements, such as color, material, light, and texture have an impact on visual perception of space ${ }^{[4]}$. A person's mood, or emotional state, and perception of experienced space are interdependent ${ }^{[5]}$. Through spatial articulation, the occupant can generate sensory-perceptive spaces with affective dimensions in order to meet his/her spatial needs. Affective spatial dimensions elicit positive or negative sensations of pleasure or displeasure and have emotional and proprioceptive impact. The perceived affective dimensions examined in this design are feelings of warmth, coolness, spaciousness, intimacy, excitement, and calmness.

The interaction is real-time, perception-based and meaning-driven. It is necessary for the system to meaningfully interpret user input, i.e., the occupant's sensory perception of affective spatial qualities. The user input-received via a slider-is subjective, unmeasurable. It is a perceived degree of an affective quality, for example, a certain degree of intimacy selected from a range between not intimate at all to extremely intimate. The corresponding spatial output is the modification of one or more relevant design parameters to articulate space. In order to provide a meaningful intent to the interaction and generate spatial output that is purposeful and effective, the design utilizes fuzzy logic for qualitative data processing structured around an underlying framework of aesthetic principles or guidelines. These aesthetic design guidelines-formulated in a previously conducted user study-are founded upon established correlations between the design parameters and perceptions of affective spatial dimensions ${ }^{[6]}$. This

1. Determine the user input and spatial output variables and establish input-output correlations based upon aesthetic guidelines.

2. Specify databases for the design parameters for the control system to access and modify surface color, material, and texture in a systematic and organized manner.

3. Employ fuzzy logic for data processing. This involves meaningfully interpret linguistic user input and generate corresponding numerical output. Fuzzy control rules are constructed from the aesthetic principles as an underlying framework.

4. Employ design scales (specific computational methods) to map the numerical output to corresponding color and material components. These components are selected to produce the final, articulated spatial output.

Figure 1 provides a conceptual diagram of the design process of the control system.

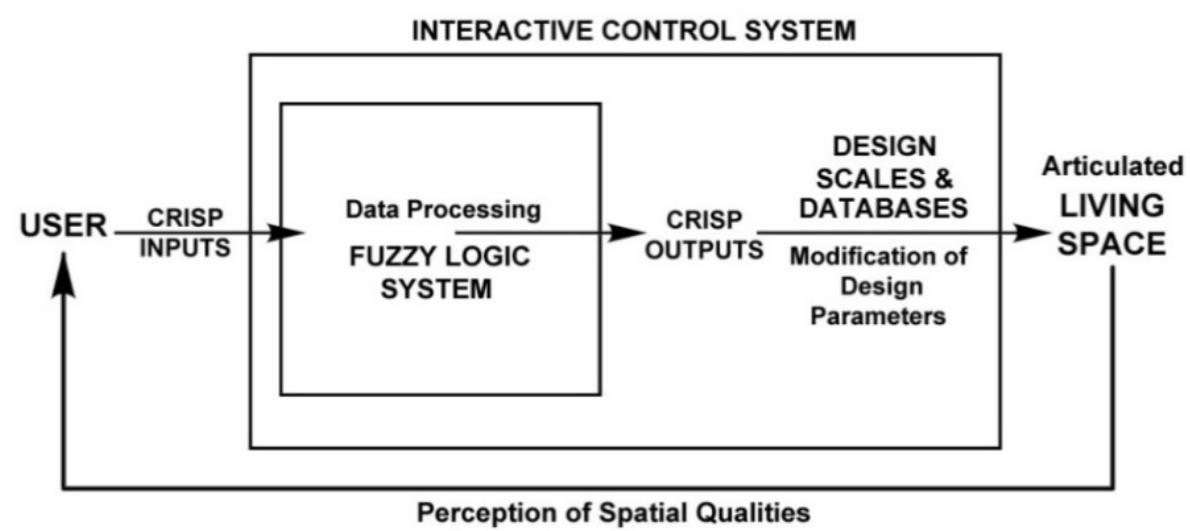

Figure 1: Conceptual Diagram of the Control System Design 


\section{OVERVIEW OF THE LIVING SPACE}

For the purpose of design, the living space is envisioned as a square-shaped, single, multi-functional living space. The concept draws its inspiration from the efficiency, studio, or "micro" apartments rented by the digital-age population living in high-density big cities, such as New York or San Francisco ${ }^{[8]}$. It is conceived as a single colored space with an accent wall that acts as a focal point of interest with modifiable material. Drywall is applied to the remaining walls and ceiling. Fabric (carpet) is applied to the floor. The size of the living space is large enough to contain a few pieces of furniture (e.g., single bed, a study table, storage case) for supporting a minimal lifestyle.

\section{FORMULATION OF THE AESTHETIC GUIDELINES}

In a previous user study conducted in a six-sided immersive Cave Automatic Virtual Environment (CAVE)-type display, the living space was simulated with adapted equivalent parameters of color, brightness, and texture (see Figure 2) with the aim of evaluating affective space creation and measuring the extent to which the design parameters influenced user's emotional responses to perceived spaces ${ }^{[6]}$. Affective spatial dimensions were examined in the following categories: temperature (warm and cool), level of arousal (exciting and calm), and size (intimate and spacious). Other dimensions examined were comfort and spatial preferences for work and rest. As perception depends on context, users were divided into two groups where one group performed an activity (active) and the other remained idle (inactive).

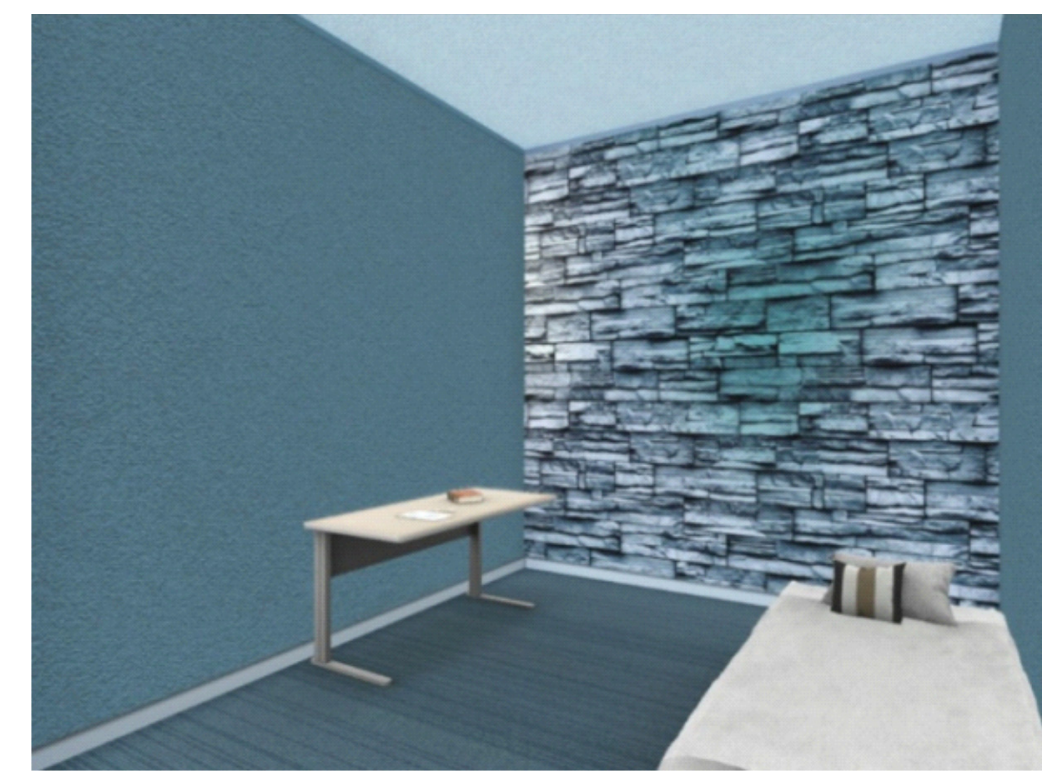

Figure 2: Simulated Living Space in a CAVE-type Virtual Reality System

Generated data confirmed the hypothesis posed in the user study that real-world impact of affective space creation could be successfully replicated in a simulated environment with adapted design parameters. Affective spatial dimensions were perceptible in the Virtual Environment (VE) and oppositional affective dimensions-i.e., warm and cool, exciting and calm-evoked contrasting emotional responses. Data pertinent to design were analyzed; both direct and inferred correlations were drawn between perception and design parameters through quantitative and qualitative data analysis. The results confirmed certain normative real-world color and brightness perceptions. As with real-world color perception, orange (perceived as a warm color) felt warmer than blue (perceived as a cool 
color). Warm colors (orange) felt more exciting and intimate than cool colors (blue). Color was found to have significant impact on perceptions of warm, cool, exciting, calm, intimate spaces. On the other hand, brightness had a significant impact on perception of spaciousness. It could be inferred from the data that texture influenced the perceived level of excitement and calmness of a space. Softness-a color perception related to visual and tactile aspects of objects-could be inferred as an affective quality having a significant impact on space perception. The visual weight of materials was inferred to have an influence on perceived spaciousness or intimacy.

A set of aesthetic principles could be articulated from derived correlations ${ }^{[6,7]}$. Table 1 presents these principles outlining how the design parameters can be modified to achieve desired affective spatial qualities. These guidelines serve as an underlying framework to construct the fuzzy control rules in order to process and interpret subjective, qualitative data meaningfully.

Table 1: The Aesthetic Guidelines ${ }^{[7]}$

\begin{tabular}{|c|c|c|c|}
\hline \multirow{9}{*}{ 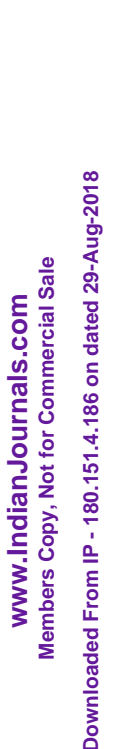 } & Desired Dimensions & Parameters to modify & Guidelines \\
\hline & Warm & Color & $\begin{array}{l}\text { Use warm colors (variations of red, orange or yellow) } \\
\text { Use warm shades of cool colors }\end{array}$ \\
\hline & Cool & Color & $\begin{array}{l}\text { Use cool colors (variations of blue, green or purple) } \\
\text { Use cool shades of warm colors }\end{array}$ \\
\hline & Spacious & $\begin{array}{l}\text { Brightness } \\
\text { Material }\end{array}$ & $\begin{array}{l}\text { Increase brightness } \\
\text { Use lighter materials }\end{array}$ \\
\hline & Intimate & $\begin{array}{l}\text { Brightness } \\
\text { Material }\end{array}$ & $\begin{array}{l}\text { Decrease Brightness } \\
\text { Use heavier materials }\end{array}$ \\
\hline & Exciting & $\begin{array}{l}\text { Texture } \\
\text { Color }\end{array}$ & $\begin{array}{l}\text { Roughen texture } \\
\text { Use stronger or more saturated colors }\end{array}$ \\
\hline & Calm & $\begin{array}{l}\text { Texture } \\
\text { Color }\end{array}$ & $\begin{array}{l}\text { Smoothen texture } \\
\text { Use muted colors }\end{array}$ \\
\hline & Muted & Color & Use muted colors \\
\hline & Saturated & Color & Use stronger or more saturated colors \\
\hline
\end{tabular}

\section{INPUT AND OUTPUT VARIABLES}

\subsection{Input Variables}

The user input is the occupant's subjective perception of affective spatial qualities. The eight input variables can be divided into four categories: 1) temperature (warm, cool), 2) size (spacious, intimate), 3) level of arousal (exciting, calm), and 4) softness (muted, saturated). In the interactive living space, qualitative user responses may be received in various forms, such as gesture and posture recognition or voice commands. The system will convert such responses into crisp input data. Such aspects-related to user interfaces-are not within the scope of design in this research.

Fuzzy logic computation is concerned with crisp input data and converts them to linguistic data. For the purpose of this paper, it is assumed that while the system receives qualitative user responses via a continuous slider scale, they are converted to corresponding crisp inputs. Figure 3 demonstrates the conceptual slider scale for the input category of level of arousal labeled with bipolar endpoints. The left end indicates the extremity of one variable and right end indicates the extremity of its contrasting variable. 


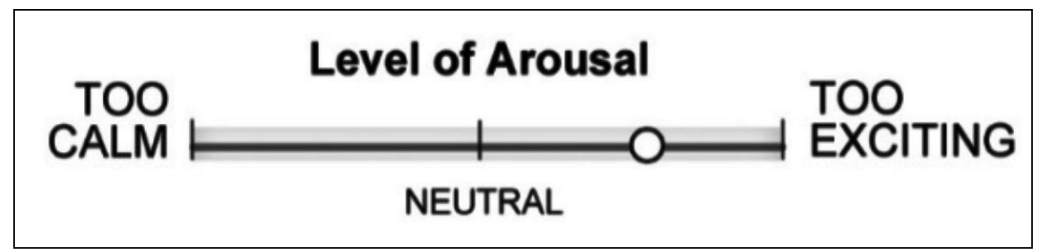

Figure 3: Concept of an Input Slider

For each category, the user moves the slider towards right or left to indicate an intermediate or extreme position on a certain statement pertaining to perception of affective spatial quality. In Figure 3, the user selects his/her perceived level of calmness or excitement for the space in relation to the two extreme positions: "too calm" on left end and "too exciting" on the right. For the purpose of fuzzy logic computation, the system converts the user selection to numerical data on a scale of 0 to 10 , where 0 is one extreme position and 10 is the other. The mid-point 5 is a neutral position.

\subsection{Output Variables}

The output variables are attributes or components of the design parameters (color, brightness, texture and material). Modifying output variables modify the appearance of the enclosing surfaces of the living space. This further impacts the perception of the overall affective qualities of the living space. There are five output variables:

1. Hue: An attribute of color. It is associated to the perceived temperature of color.

2. Value: An attribute of color. It indicates the level of brightness or darkness of a color.

3. Chroma: An attribute of color. It indicates the intensity (saturation) of a color.

4. Graininess: An attribute of texture. It indicates the level of roughness or smoothness of a texture.

5. Material type: It indicates the type of a material, such as wood, stone, or fabric

Table 2 lists the design parameters, the associated output variables and the corresponding enclosing surfaces of the living space that these variables can modify.

Table 2: Output Variables and Associated Modifiable Surfaces

\begin{tabular}{llll}
\hline Design parameters & \multicolumn{2}{l}{ Output Variables (Surface Attributes) } & Modify Surfaces \\
\hline Color & Hue & Name \& Temperature & All \\
& Chroma & Intensity or Saturation & All \\
Brightness & Value & Lightness & All \\
Texture & Graininess & Rough or Smoothness & All \\
Material & Material type & Type & Accent wall \\
\hline
\end{tabular}

\section{SPECIFYINGDATABASESFOR COLOR, TEXTURE AND MATERIAL}

The surface color, brightness, texture and material are modified as an outcome of interaction. In order to do so, the control system is assigned color, texture and material databases. The system uses these databases to map the output data and find corresponding hue, chroma, value, graininess, and material type.

The color database is a three-dimensional color space similar to the traditional Munsell color system ${ }^{[9]}$. The color space consists of hue, value and chroma spectrums. The hue spectrum consists of twenty hues (colors) convered 
into a band of continuous, smoothly changing gradient. Warm and cool hues are arranged according to their color temperature. Warmth or coolness of a hue depends on its relative distance from pure red (RGB \#ff0000) as the warmest hue or pure blue (RGB \#0000ff) as the coolest hue ${ }^{[9]}$. The value spectrum is a band of continuous brightness gradient for any given color from pure black (0\%) to pure white (100\%). The chroma spectrum, for any given color, is a band of continuous gradient from grey to its full saturation or intensity.

The texture database consists of ranges of texture graininess. These are available for textures of five materialsstone, wood, fabric (curtain), fabric (carpet) and drywall-ranging from very smooth to very rough. The material database for the control system consists of three materials specified for the accent wall: stone, wood, and fabric (curtain). Based upon output data, the control system selects a material to replace the existing material for only the accent wall.

\section{ESTABLISH INPUT-OUTPUT CORRELATIONS}

The aesthetic guidelines can be expanded to include the input and output variables in order to establish correlations between them, as shown in Table 3. Each input variable is related to one or more output variables to modify in order

Table 3: Input-Output Correlations from Aesthetic Guidelines

\begin{tabular}{|c|c|c|c|}
\hline \multicolumn{2}{|c|}{ Input } & \multicolumn{2}{|c|}{ Output } \\
\hline Perceptual Categories & Input Variables & Parameters to Modify & Output variables \\
\hline Temperature & Warm and Cool & Color & Hue \\
\hline \multirow[t]{2}{*}{ Size } & Spacious and Intimate & Brightness & Value \\
\hline & & Material & Type \\
\hline \multirow[t]{2}{*}{ Level of Arousal } & Calm and Exciting & Texture & Graininess \\
\hline & & Color & Chroma \\
\hline Softness & Muted and Saturated & Color & Chroma \\
\hline
\end{tabular}

The following sub-sections explain how each affective spatial dimension can be modified through manipulation of one or more design parameter attributes on the basis of input-output correlations established.

\subsection{Modify Perceived Warmth or Coolness}

The perceived temperature (warmth or coolness) of the living space can be modified by manipulating hue for all enclosing surfaces. A warmer hue increases perceived warmth while a cooler hue increases perceived coolness of the space. In order to increase the level of warmth or coolness perceived by the user, the control system shifts along the hue spectrum of the color database to select a hue that is warmer or cooler than the existing surface hue respectively (Figure 4a).

\subsection{Modify Perceived Spaciousness or Intimacy}

The perceived size of the living space can be modified by changing color value and material type for all enclosing surfaces. The value component of surface color controls its perceived brightness. A higher value darkens the color and enhances perceived intimacy, whereas a lower value lightens the color and enhances perceived 


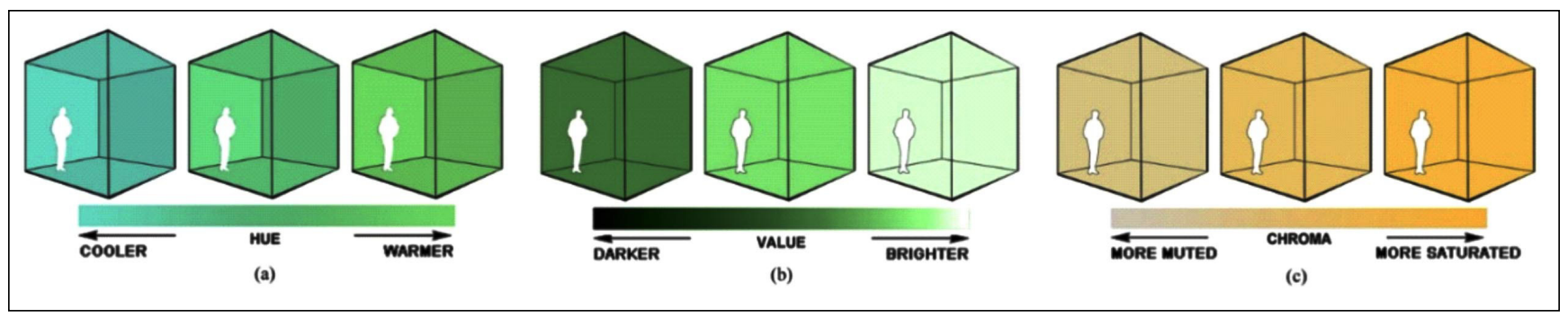

Figure 4: Modification of perceived affective spatial qualities. (a) changing surface hue impacts perceived temperature; (b) changing surface color value impacts perceived size; (c) changing surface color chroma impacts perceived level of arousal and softness

spaciousness. In order to increase the degree of spaciousness or intimacy perceived by the user, the control system shifts along the value spectrum of the color database to select a value that is higher or lower than the existing surface color value respectively (Figure 4b).

In addition to the color value, material type is also modified for the accent wall to enhance perceived spaciousness or intimacy. The perception of rigidity, confinement, openness, spaciousness, intimacy, or lightness of a space is

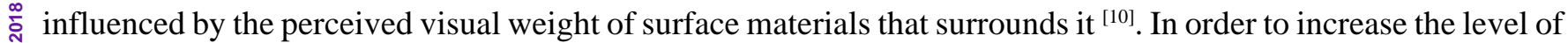
spaciousness or intimacy of space, the control system selects a material for the accent wall from the material database that is visually lighter or heavier than the existing material respectively.

\subsection{Modify Perceived Excitement or Calmness}

The perceived level of arousal of the living space is modified by changing graininess and chroma for all enclosing surfaces. Graininess of surface texture has an impact on the perceived excitement or calmness of a space. Rougher texture enhances perceived level of excitement, while smoother texture enhances perceived level of calmness. In order to modify the level of excitement or calmness of space perceived by the user, the control system changes the level of texture graininess for each existing surface texture.

In addition to texture graininess, surface chroma is also modified. A higher chroma creates a stronger, more saturated surface color and increases the perceived degree of excitement. On the other hand, a lower chroma creates a softer, less saturated or muted color for the surfaces and increases the perceived degree of calmness of the space. The control system shifts along the chroma spectrum of the color database to select a chroma that is higher or a lower than that of the existing surface color (Figure 4c).

\subsection{Modify Perceived Softness}

Muted and saturated are affective aspects of color. Perceived softness of a space can be controlled by changing the chroma component of surface color. Muted or low saturated colors increase visual softness of space, while saturated colors create contrasting effect. For the living space, the control system utilizes the chroma spectrum of the color database to select a chroma higher or lower than that of the existing surface color to create a more saturated or a more muted spatial quality respectively (Figure $4 \mathrm{c}$ ).

\section{APPLICATION OF FUZZY LOGIC SYSTEM}

Fuzzy logic is a soft computational method implemented in control system design for analyzing soft, qualitative (fuzzy), linguistic data pertaining to abstract concepts and statistical ambiguity arising from contradictory or complex 
situations ${ }^{[11]}$. In fuzzy logic, interpretation is driven by inferences, intuitive or implied meanings, as well as pattern recognition. Fuzzy logic has been successfully applied in architectural designs where decision-making not only involves quantitative data analysis, but also revolves around abstract ideas and non-numerical data ${ }^{[12]}$. Although not regularly implemented, fuzzy logic has been accepted as an appropriate mathematical model for architectural design to process non-quantifiable data pertaining to subjective human perceptions, behavior, opinions, experiences and lifestyles ${ }^{[13]}$.

In this paper, design of human-space interaction is structured around subjective perception of affective or emotional spatial qualities. User responses that pertain to perceptions of emotional qualities of space-i.e., how warm, cold, exciting, calm, spacious, or intimate a space feels-cannot be represented as classical data of absolute true (1) or false (0). For instance, a space can be verbally described as very spacious, quite spacious or a little intimate. Expressions such as very, quite, moderately or a little are non-quantifiable. In addition to that, the space may also feel both spacious and slightly intimate to a certain degree. In order toanalyse and interpret such linguistic data in a way that is meaningful to the user, fuzzy logic is employed to introduce the idea of partial truth in data processing. Fuzzy logic draws inferences to represent data in terms of degrees of association or membership. In order to represent a specific size of a space as both partially spacious and intimate, fuzzy logic formulates membership $\stackrel{\infty}{5}$ functions-in the form of graphical representations of linguistic variables-to assess the transition between spacious

In the design of the control system, the processing of input and output data in fuzzy modeling is conducted in three parts as illustrated in Figure 5. The required steps are as follows:

1. Fuzzification: Membership functions are defined for both input and output variables in order to convert the slider input received from the user into meaningful linguistic data with fuzzy components to varying degrees.

2. Fuzzy Inference: Fuzzy control rules-a set of IF-THEN conditions founded upon the aesthetic principlesare applied to regulate meaningful input-output relations and derive linguistic outputs.

3. Defuzzification: The linguistic outputs are converted or defuzzified into numerical outputs through specific computational method.

The Fuzzy Logic Toolbox of MATLAB, a computing software, is utilized to design the graphical membership functions, apply the fuzzy rules and compute the final numerical outputs in the defuzzification process presented in this paper.

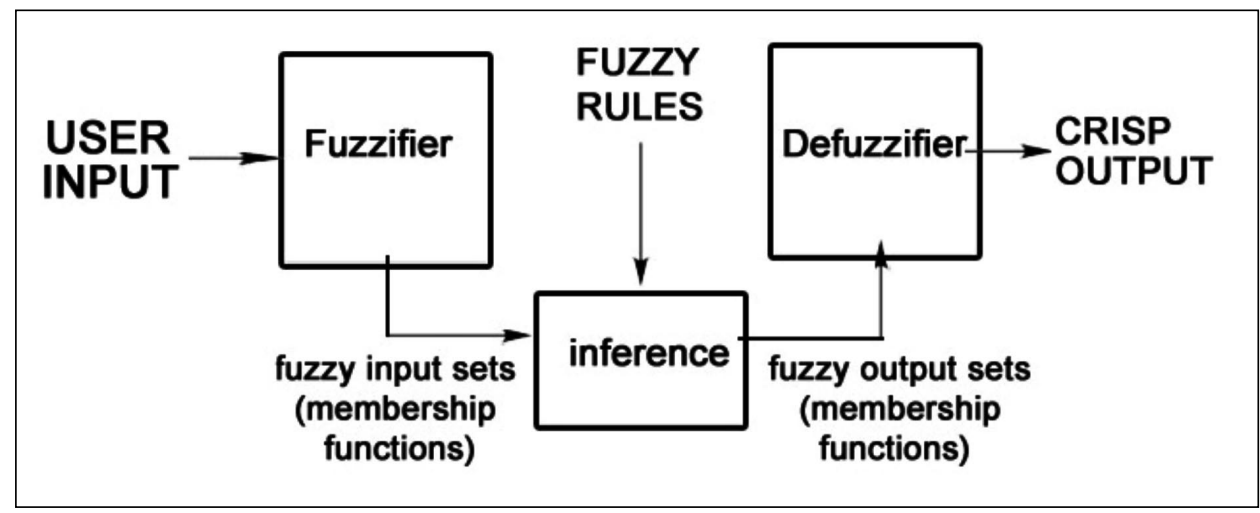

Figure 5: Conceptual Diagram of Fuzzy Logic Data Processing 


\section{FUZZIFICATION: DEFINING MEMBERSHIP FUNCTIONS}

In the fuzzification process, membership functions for both input and output variables are defined and converted to linguistic variables. The membership functions are graphical representations in the form of linguistic variables or fuzzy data sets with numerical ranges. The purpose of these visual representations is to ascribe degrees of membership (DOM) to each input and output variable through linear interpolation.

\subsection{Defining Input Membership Functions}

Triangular membership functions are utilized to represent the four input categories of temperature, size, level of arousal and softness each with a set of five linguistic variables. The linguistic input variables (fuzzy inputs) of each category are listed in Table 4 . All input membership functions have been set within a boundary ranging from 0 to 10 units. For each membership function, the range is divided into five sub-ranges for the five linguistic variables. The centers of the five linguistic variables are equidistant from each other, located at 0,2.5, 5, 7.5, and 10. Table 5 shows the numerical ranges of input linguistic variables for the input membership function of size. Figure 6 shows the triangular wave form for input membership function for size. The membership functions of temperature,

Table 5: Ranges of Input Linguistic Variables of Size

\begin{tabular}{ll}
\hline Input Linguistic variables & Range of Crisp User Input \\
\hline Too intimate & Less than 0 to +2.5, with 0 as the center \\
Slightly intimate & 0 to +5, with 2.5 as the center \\
Neutral & +2.5 to +7.5, with 5 as the center \\
Slightly spacious & +5 to +10, with 7.5 as the center \\
Too spacious & +7.5 to more than +10, with 10 as the center
\end{tabular}

The control system converts the user inputs into corresponding crisp inputs. Computation of degree of membership for each input variable is conducted through linear interpolation by mapping the values between 0 to 1 on the vertical axis as illustrated in Figure 6. For each crisp input, the corresponding membership values $(\mu)$ for each linguistic variable (fuzzy set) are calculated. This means, for the crisp input of size $=1$, it belongs to: "too intimate" to 0.6 degree, "slightly intimate" to 0.4 degree, "neutral" to 0 degree, "slightly spacious" to 0 degree, and "too spacious" to 0 degree.

\subsection{Defining Output Membership Functions}

Both triangular and trapezoid membership functions have been used to represent the five output variables-hue, chroma, value, graininess \& material type. Each consists of a set of five linguistic variables presented in Table 6. 


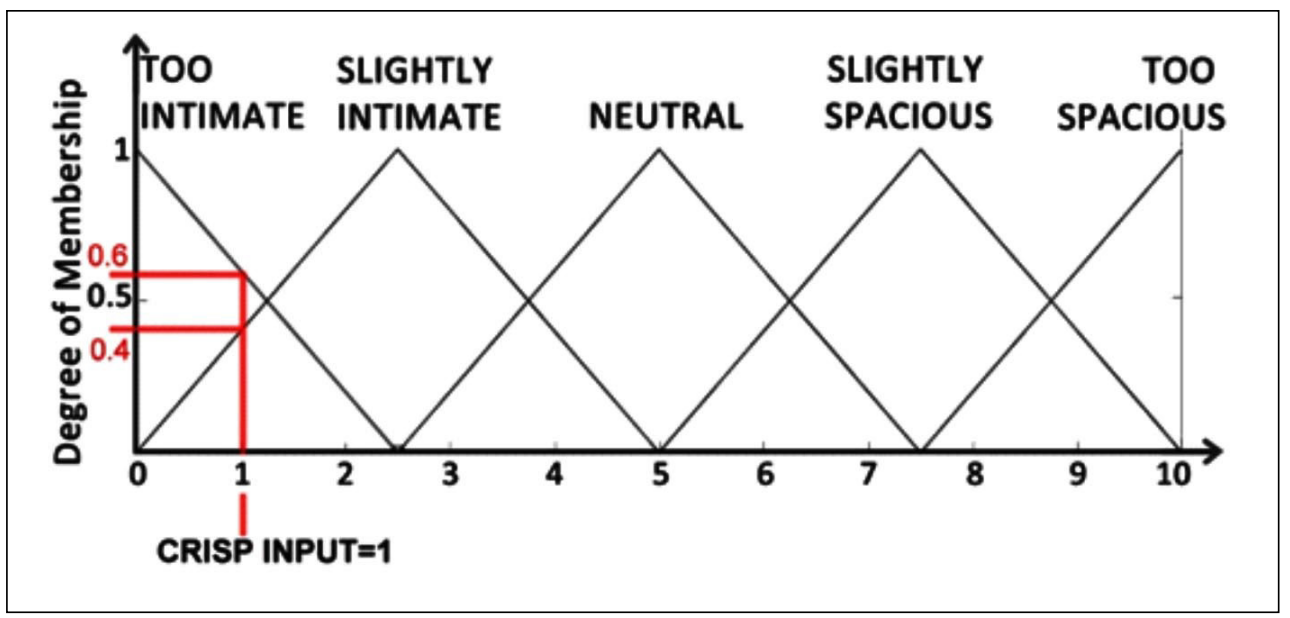

Figure 6: Triangular Waveform for Input Membership Function for Size

Table 6: Output Linguistic Variables

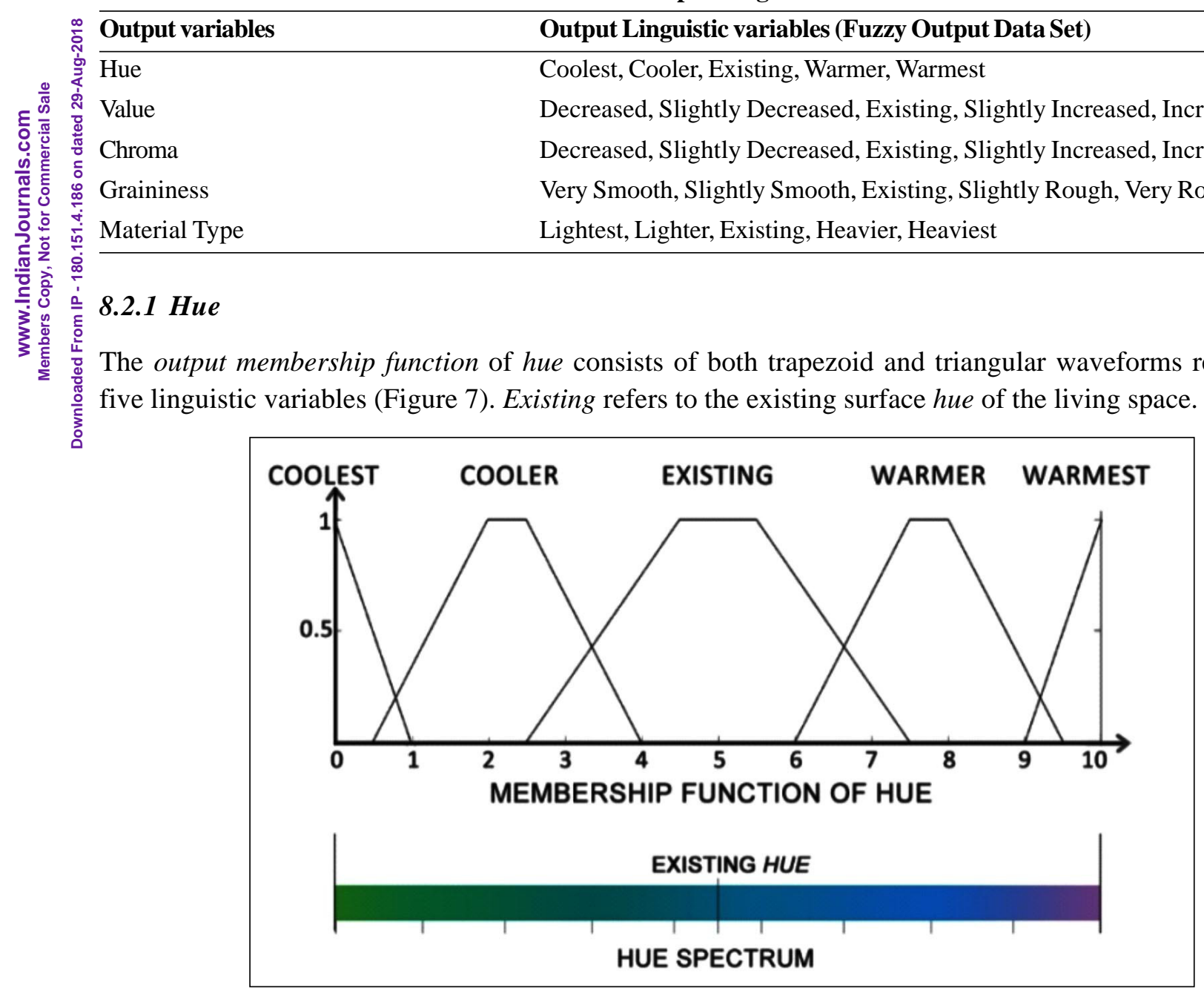

Figure 7: Output membership function for Hue 
The waveforms within the 0-10 range are designed to correspond to a segment of the hue spectrum with the existing surface hue located at the center. The length of the segment equals to the length of any ten consecutive hues of the Munsell color system ${ }^{[9]}$. The specific length is selected to ensure a large variation of hues within the 0 -10 range. Table 7 presents the ranges of each linguistic variables.

Table 7: Hue: Ranges of Output Linguistic Variables

\begin{tabular}{ll}
\hline Linguistic variables & Range of Crisp Output \\
\hline Coolest & Less than 0 to +1, with 0 as the center \\
Cooler & +0.5 to +4, with 2.25 as the center \\
Existing & +2.5 to +7.5, with 5 as the center \\
Warmer & +6 to +9.5, with 7.75 as the center \\
Warmest & +9 to more than +10, with 10 as center \\
\hline
\end{tabular}

\subsubsection{Value and Chroma}

The output membership functions of both value and chroma are represented by trapezoid waveforms of five

For the membership function of chroma, the waveforms correspond to a subset of the chroma spectrum with the existing surface chroma located at the center (Figure 8). For any given hue, its chroma spectrum consists of 1unit increments in chroma. To ensure a large variation of color intensity, the length of the subset selected here consists of four units of chroma.

\subsubsection{Graininess}

The output membership function of graininess is also represented by trapezoid waveforms of five linguistic variables within a range of 0 to 10 (Figure 9). Existing refers to the existing graininess of the surface texture. The waveforms within the range are designed to correspond to a specific segment of the texture gradient with the existing surface texture graininess located at the center. The segment consists of five variations of graininess in $10 \%$ increments. The ranges of linguistic variables are the same as that of value and chroma linguistic variables. 


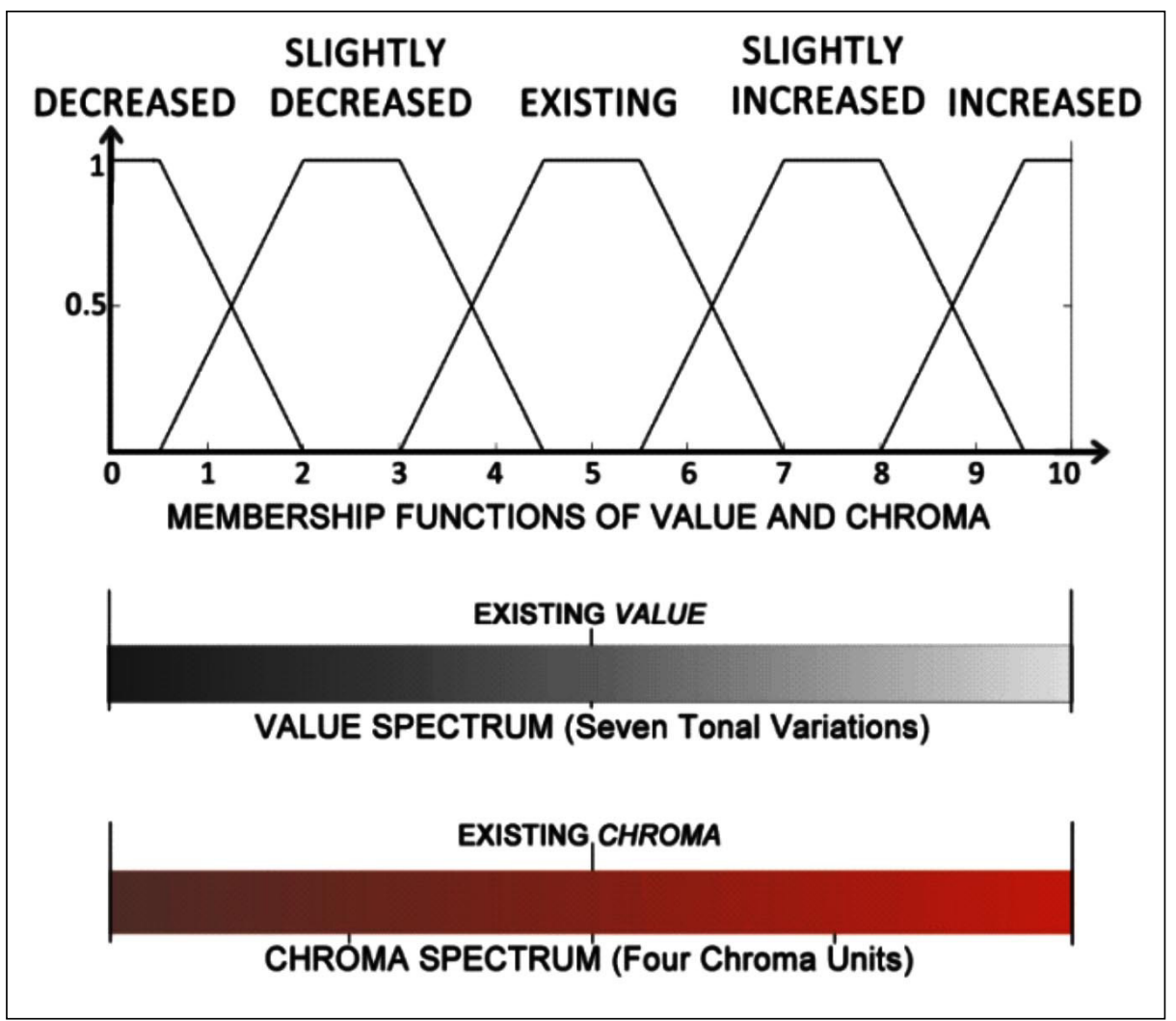

Figure 8: Output membership function for Value and Chroma

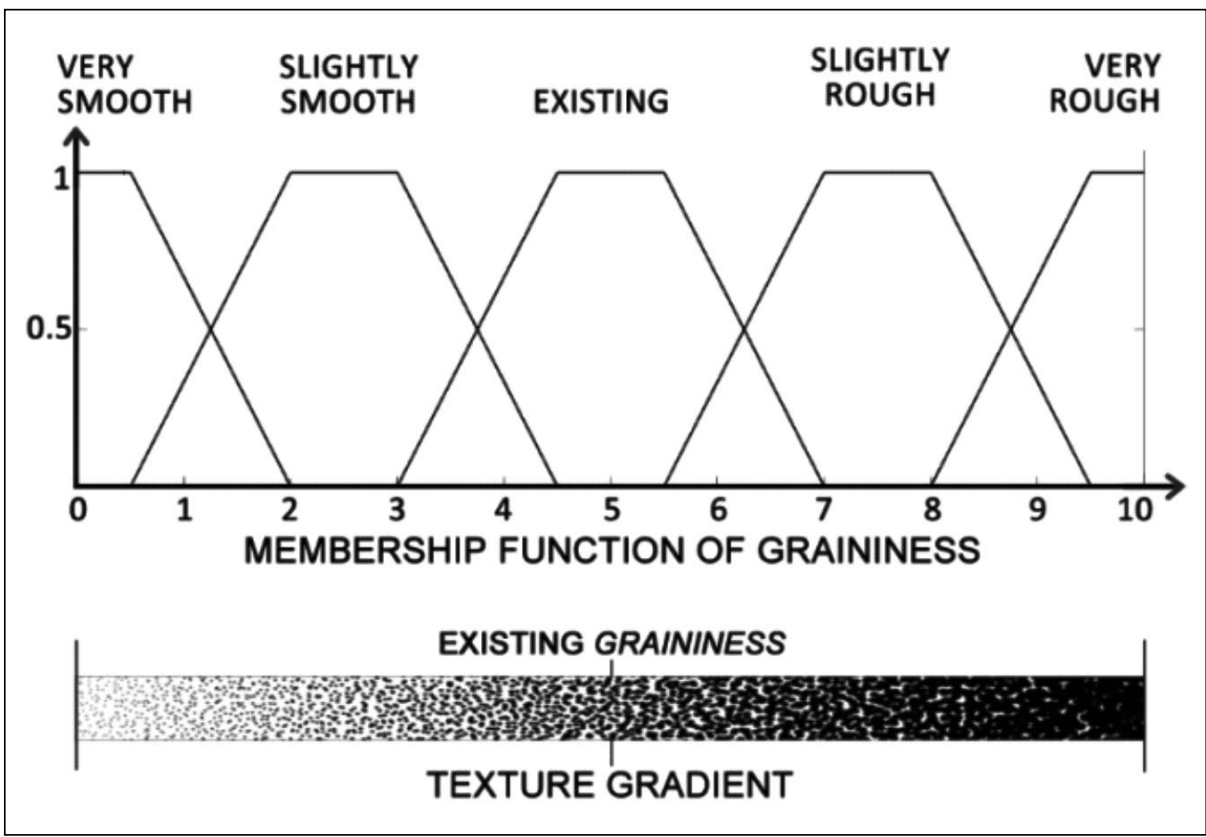

Figure 9: Output membership function for Graininess 


\subsubsection{Material Type}

The output membership function of material type is represented by triangular waveforms of five linguistic variables within a range of 0 to 10 (Figure 10). Existing refers to the existing surface material. Each linguistic variable indicates a material for the accent wall selected on the basis of their perceived visual weight. Table 9 presents the ranges of each linguistic variable.

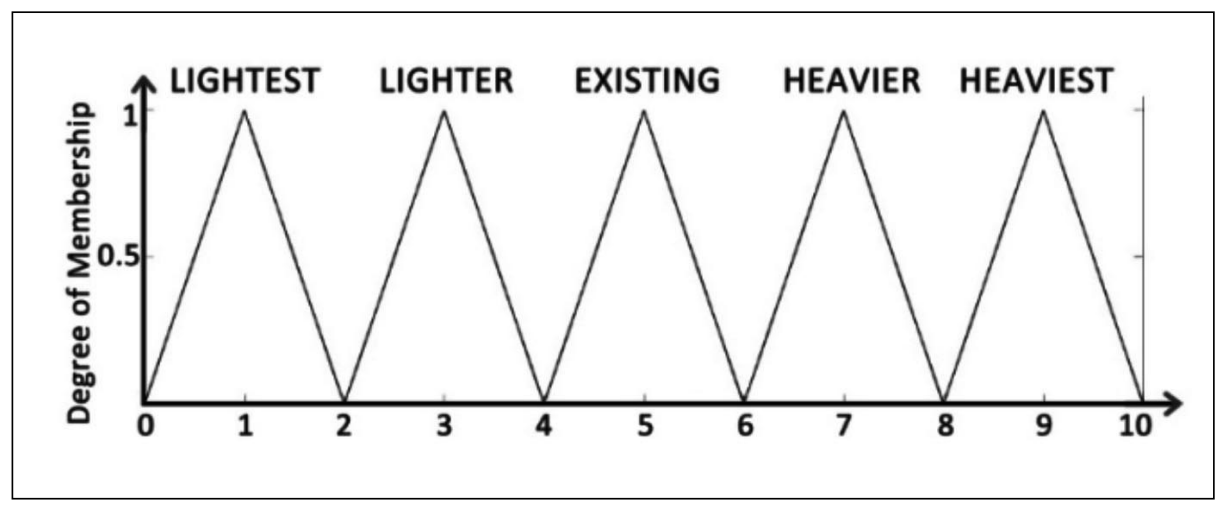

Figure 10: Output membership function for Material Type

Table 9: Material Type: Ranges of Output Linguistic Variables

\begin{tabular}{ll}
\hline Linguistic variables & Range of Crisp User Output \\
\hline Lightest & 0 to +2, with 1 as the center \\
Lighter & +2 to +4, with 3 as the center \\
Existing & +4 to +6, with 5 as the center \\
Heavier & +6 to +8, with 7 as the center \\
Heaviest & +8 to +10, with 9 as the center \\
\hline
\end{tabular}

\section{AESTHETIC PRINCIPLES AS FUZZY CONTROL RULES}

Fuzzy control rules are constructed from input-output correlations established on the basis of aesthetic principles (see Table 3). The rules provide meaning to data processing and applied once the input and output variables are fuzzified. Each rule is a linguistic IF-THEN condition that combines input and output membership functions in order to control how the system will respond to each user input when certain conditions are met. The IF-command pertains to a condition of input variable. The THEN-command is the action the system takes if one or more IFconditions are met. In the fuzzy inference process, the input categories (temperature, size, level of arousal, and softness) and output variables (hue, value, chroma, graininess, and material type) are combined on the basis of the following fuzzy rules:

Rules for Membership Function for Temperature:

1. IF (Temperature is too cold) THEN (Hue is Warmest)

2. IF (Temperature is slightly cold) THEN (Hue is Warmer)

3. IF (Temperature is neutral) THEN (Hue is Existing)

4. IF (Temperature is slightly warm) THEN (Hue is Cooler)

5. IF (Temperature is too warm) THEN (Hue is Coolest) 
Rules for Membership Function for Size:

1. IF (Size is too intimate) THEN (Value is Increased) AND (Material Type is Lightest)

2. IF (Size is slightly intimate) THEN (Value is Slightly Increased) AND (Material Type is Lighter)

3. IF (Size is neutral) THEN (Value is Existing) AND (Material Type is Existing)

4. IF (Size is slightly spacious) THEN (Value is Slightly Decreased) AND (Material Type is Heavier)

5. IF (Size is too spacious) THEN (Value is Decreased) AND (Material Type is Heaviest)

Rules for Membership Function for Level of Arousal:

1. IF (Level of Arousal is too calm) THEN (Graininess is Very Rough) AND (Chroma is Increased)

2. IF (Level of Arousal is slightly calm) THEN (Graininess is Slightly Rough) AND (Chroma is Slightly Increased)

3. IF (Level of Arousal is neutral) THEN (Graininess is Existing) AND (Chroma is Existing)

4. IF (Level of Arousal is slightly exciting) THEN (Graininess is Slightly Smooth) AND (Chroma is Slightly Decreased)

5. IF (Level of Arousal is too exciting) THEN (Graininess is Very Smooth) AND (Chroma is Decreased)

Rules for Membership Function for Softness:

1. IF (Softness is too muted) THEN (Chroma is Increased)

2. IF (Softness is slightly muted) THEN (Chroma is Slightly Increased)

3. IF (Softness is neutral) THEN (Chroma is Existing)

4. IF (Softness is slightly saturated) THEN (Chroma is Slightly Decreased)

5. IF (Softness is too saturated) THEN (Chroma is Decreased)

\section{DEFUZZIFICATION: CRISP OUTPUT DATA}

The fuzzy control rules are applied to the linguistic input and output membership functions to produce the final crisp outputs. The MATLAB Fuzzy Logic Toolbox is used defuzzfication calculations. The centroid method-a popularly used method to compute the center of gravity of combined linguistic outputs-is implemented to produce the aggregated crisp output. Figure 11 illustrates-through graphical representations-several scenarios of possible user inputs that generate corresponding defuzzified crisp outputs. The scenarios are explained below:

1. Scenario 1: When user perceives the space as very warm (temperature $=9.6)$, the system changes surface color to a very cool hue (hue $=1.3$ ) in order to make the space feel cooler.

2. Scenario 2: When user perceives the space as extremely intimate (size=1), the system increases the surface brightness substantiall (value $=8.12$ ) and changes the material for the accent wall to a visually light-weight material (material type $=1.86$ ) in order to make the space feel more spacious.

3. Scenario 3: When user perceives the space as somewhat calming (level of arousal = 3.17), the system slightly increases the intensity of the surface color (chroma $=6.14)$ as well as the roughness of the surface texture (graininess $=6.77$ ) in order to make it feel more exciting. 

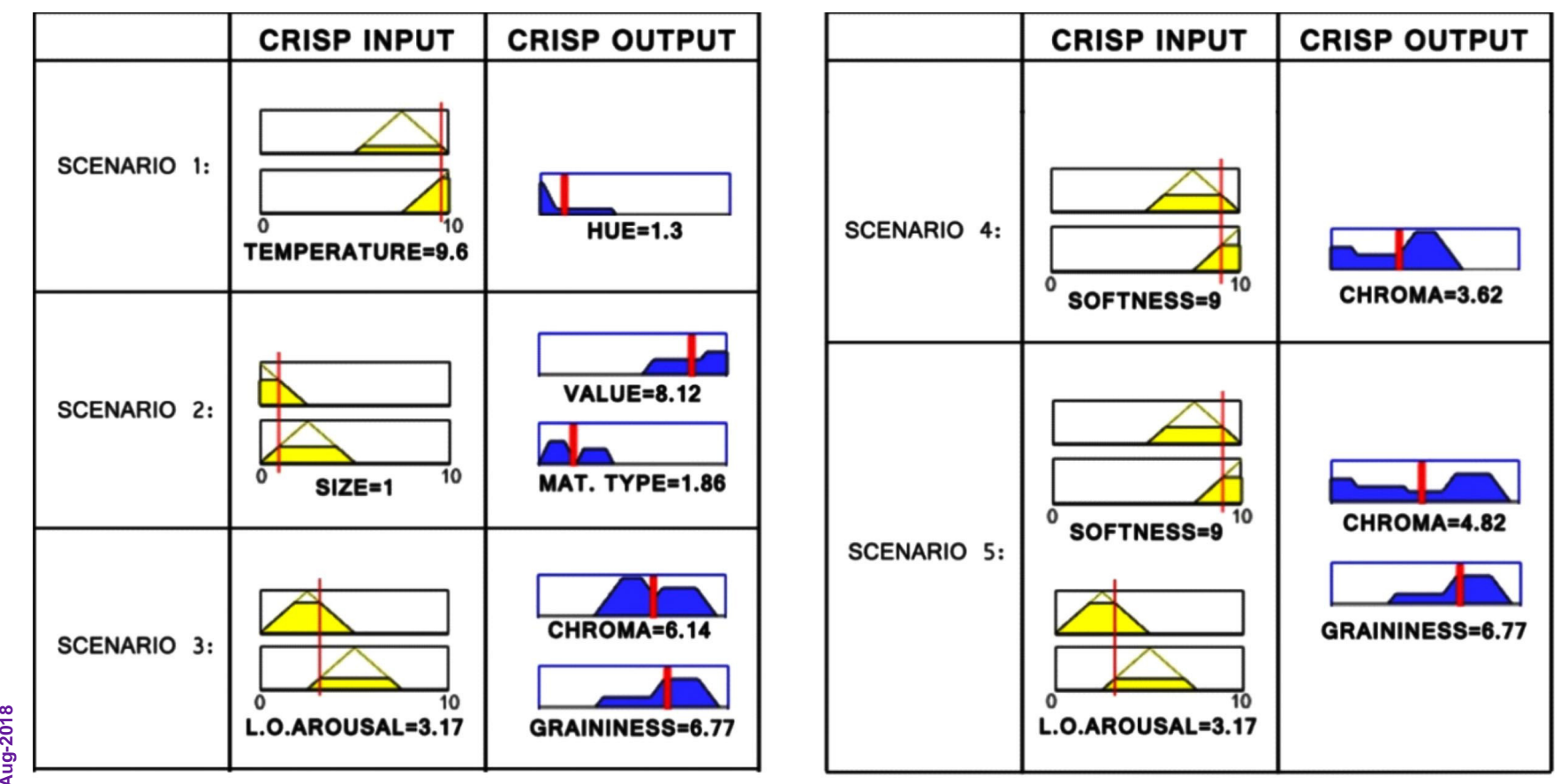

Figure 11: Some scenarios presenting graphical representations of crisp inputs and corresponding defuzzifiedcrisp outputs

4. Scenario 4: When user perceives the space as too saturated in color (softness $=9$ ), the system decreases the intensity of surface color (chroma $=3.62$ ) to make it appear more soft and muted.

5. Scenario 5: When user perceives the space as be somewhat calming (level of arousal =3.17) but too strong or saturated in color ( softness $=9$ ), the system slightly decreases the intensity of surface color (chroma $=4.82$ ) and increases the roughness of surface texture (graininess $=6.77$ ) to make the space appear more exciting yet soft and muted.

\section{MAPPING CRISP OUTPUT TO FIND NEW SURFACE ATTRIBUTES FOR SPACE ARTICULATION}

The defuzzified crisp outputs are the final outcome of fuzzy logic data-processing. Specific computation methods are applied by the control system to find the new (i.e., modified) surface attributes-hue, value, chroma, graininess or material type that corresponds to the crisp outputs. The following sub-sections explain the process of how each crisp output is converted to its corresponding new hue, chroma, value, graininess or material type in order to produce the final, articulated spatial output.

\subsection{Finding New Surface Hue}

The defuzzified crisp output data (hue =1.3) is mapped on a segment of the hue spectrum in order to find the new color (hue), as illustrated in Figure 12. The control system selects a specific segment of the hue spectrum from the color database with the existing surface hue located at its center. The length of the segment is specified (see section 8.2.1) and corresponds to the 0-10 range of the aggregated output membership function of hue. The RGB value of the new hue is measured to replace the existing surface hue. 


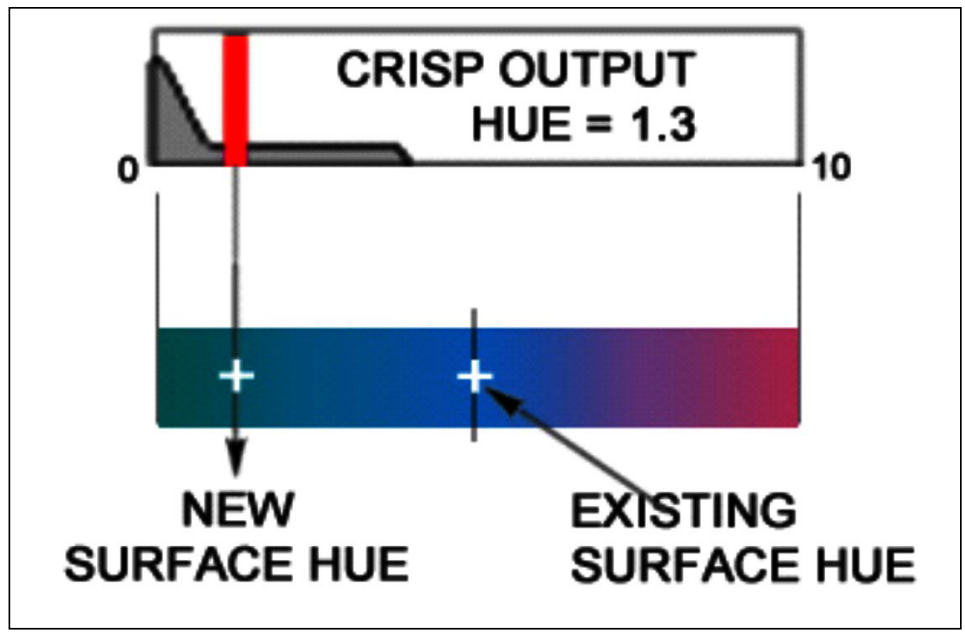

Figure 12: Mapping of crisp output to find new surface color (hue)

\subsection{Finding New Surface Value (Brightness)}

The defuzzified crisp output data (value $=8.12$ ) is mapped on a specific segment of the value spectrum to find the new value for the surface color, as illustrated in Figure 13a. The specific segment of the value spectrum is selected from the color database with the existing value of surface color located at the center. The length of the segment is specified with $30 \%$ darker value on one end and $30 \%$ lighter value on the other (see section 8.2.2). The segment corresponds to the 0 to 10 range of the aggregated output membership function of value. The control system measures the new value (brightness or darkness level) to replace the existing value of surface color.

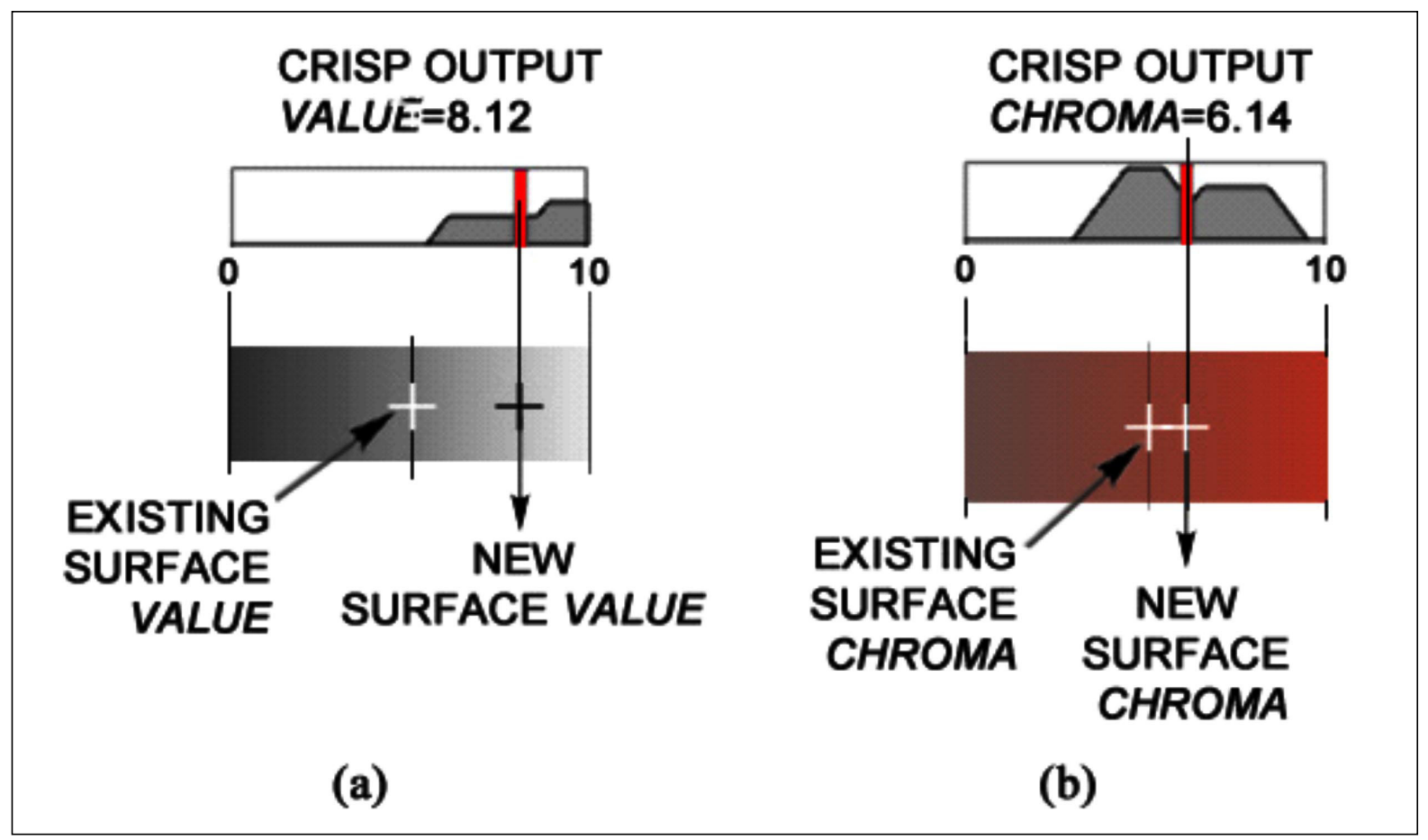

Figure 13: Mapping of crisp output to find: (a) New surface brightness (b) New surface chroma 


\subsection{Finding New Surface Chroma}

A new surface chroma can be found-as illustrated in Figure 13b-by mapping the crisp output data (chroma $=$ 6.14) on a segment of the chroma segment from the color database. The system selects a specific segment of the chroma spectrum with the existing surface chroma located at the center. The length of this segment is specified (see section 8.2.2) and corresponds to the 0 to 10 range of the aggregated output membership function of chroma. The new chroma (saturation level) is measured to replace the existing surface chroma.

\subsection{Finding New Surface Texture Graininess}

A new surface texture graininess can be found-as illustrated in Figure 14 by mapping the crisp output data (graininess $=6.77$ ) on a texture segment. From the texture database, the control system selects a segment of the texture gradient with the existing surface texture graininess located at the center. The length of the segment is specified (see section 8.2.3) and corresponds to the 0 to 10 range of the aggregated output membership function of graininess. The control system measures new graininess (roughness or smoothness level) to replace the existing surface texture graininess.

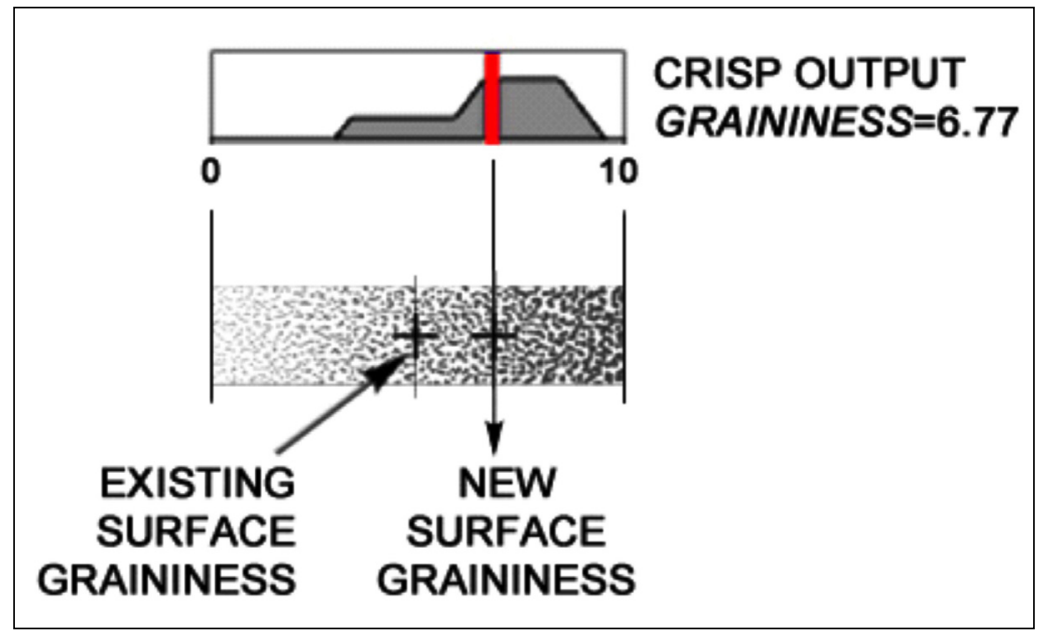

Figure 14: Mapping of crisp output to find new surface chroma

\subsection{Finding New Material for Accent Wall}

Selection of the new material for the accent wall is dependent upon its perceived visual weight relative to that of the existing material as shown in Table 10. Fabric is the lightest material and stone is the heaviest. To find the new material, the crisp output of material type is mapped on the graphical representation of the output membership function of material type as shown in Figure 15. Mapping of the crisp output data (material type $=1.86$ ) indicates the lightest material (fabric) as the new material. If crisp output points to a "heavier" material, the control system will replace fabric (curtain) with wood or replace wood with stone. If output data suggests a new material that is heavier than stone or lighter than fabric, the surface material of the accent wall will remain unchanged.

\section{DESIGN CONDITIONS AND CONSTRAINTS}

Some special design conditions have been formulated to address specific constraints or uncertainties that may emerge during human-space interaction. They are as follows: 


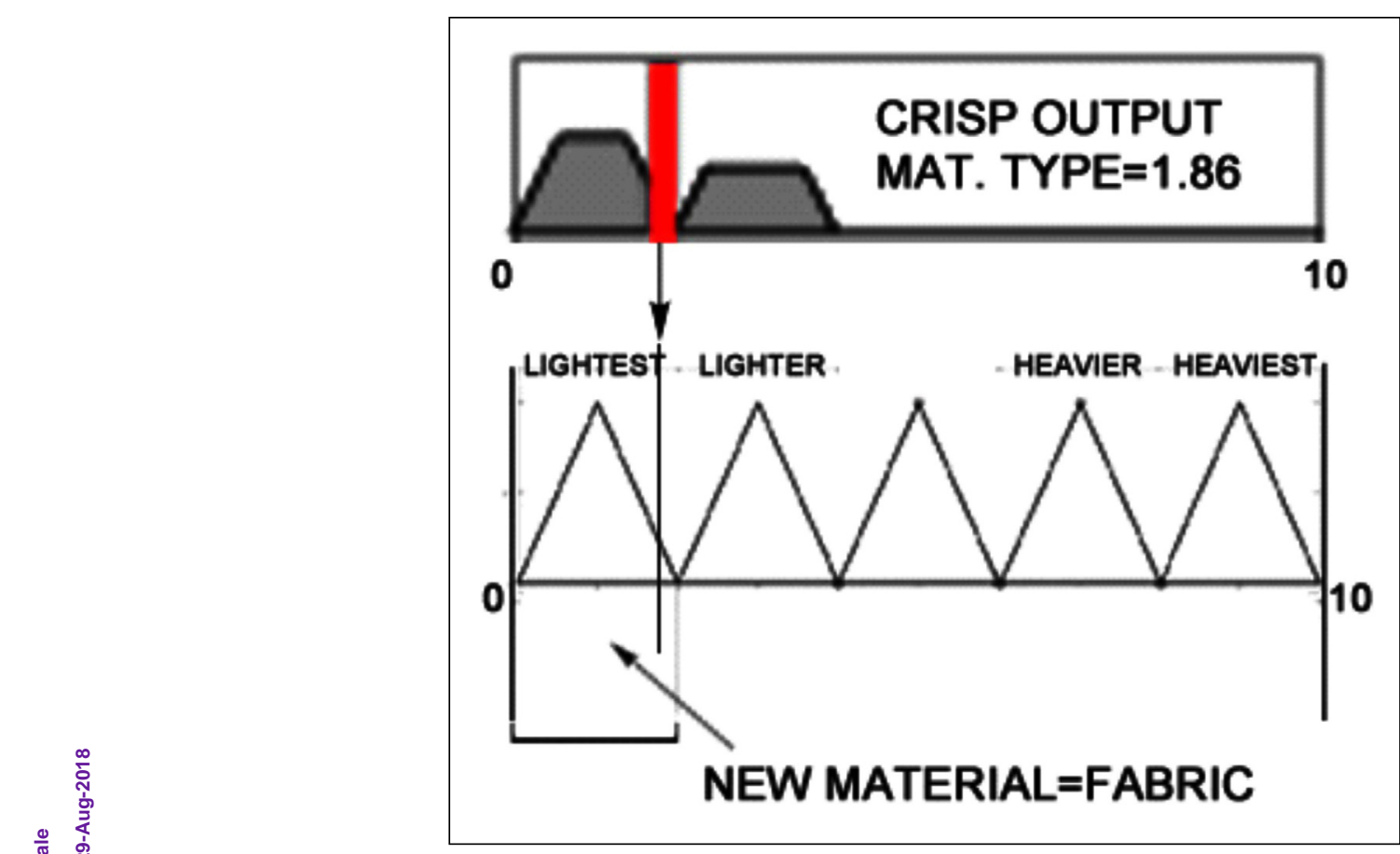

Figure 15: Mapping of crisp output to find new surface material

Table 10: Material Type: Ranges of Output Linguistic Variables

\begin{tabular}{lcccc}
\hline Existing material for & \multicolumn{4}{c}{ New Material } \\
\cline { 2 - 5 } accent wall & Lighter & Lightest & Heavier & Heaviest \\
\hline Fabric & - & - & Wood & Stone \\
Wood & Fabric & Fabric & Stone & Stone \\
Stone & Wood & Fabric & - & - \\
\hline
\end{tabular}

\subsection{Condition 1: Constraint with Surface Color}

The color database has two color modes each having pure blue (RGB \#0000FF) on one end as the coolest hue and pure red (RGB \#ff0000) on the other end as the warmest. Mode-1 consists of shades of yellow, green and orange. Mode- 2 is the relatively shorter segment that consists of shades of purple and magenta. The system uses any one color mode at a time. If the surface hue becomes absolute black, white or any shades of gray, the system will switch the color modes for any further interactions that pertains to surface color modifications.

1. When switched to mode-1, the new surface hue will be pure yellow (5Y) color at its highest level of chroma and $50 \%$ color value.

2. When switched to mode-2, the new surface hue will be pure violet (5P) at its highest level of chroma and 50\% color value.

\subsection{Condition 2: Constraints with Temperature}

Two possible scenarios may arise during manipulating perceived warmth or coolness of space: 
1. If user requests a warmer space when surface hue is already pure red (the warmest hue), the system will darken the surface color by lowering its value while keeping the hue intact.

2. If user requests a cooler space when surface hue is already pure blue (the coolest hue), the system will brighten the surface color by increasing its value while keeping the hue intact.

\section{EXAMPLES OF POSSIBLE OUTCOMES OF HUMAN-SPACE INTERACTIONS}

This section demonstrates the application of fuzzy logic in interaction data processing and presents some scenarios as possible spatial outputs in response to a range of user inputs. Crisp user inputs (on a scale of 0 to 10) have been taken into account for the demonstration. Here, 0 indicates the extremity of one input variable and 10 indicates the extremity of its contrasting variable (e.g., warm and cool). MATLAB is used to conduct fuzzy logic computations. The defuzzified crisp outputs are then mapped to appropriate databases to find the newly modified color, brightness, texture and/or material for the articulated living space. Computer-rendered images (Figures 17-20) display the articulated spatial outputs. Two existing conditions (Figure 16) have been selected for the living space: 1) Space-A: Green surface color (cool hue) with a stone accent wall and 2) Space-B: Yellow surface color (warm hue) with a

\section{Figure 16: Two existing environments for the living} space

Figure 17: Spatial outputs generated for Temperature inputs

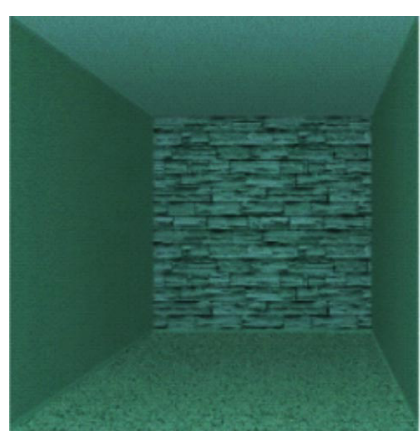

SPACE-A

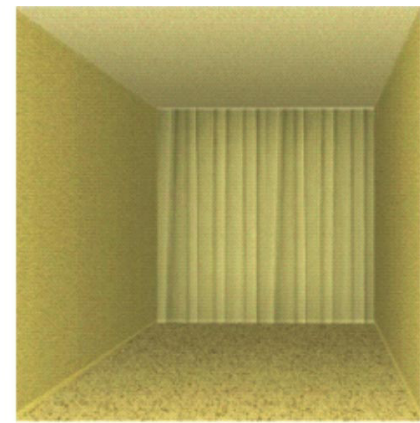

SPACE-B

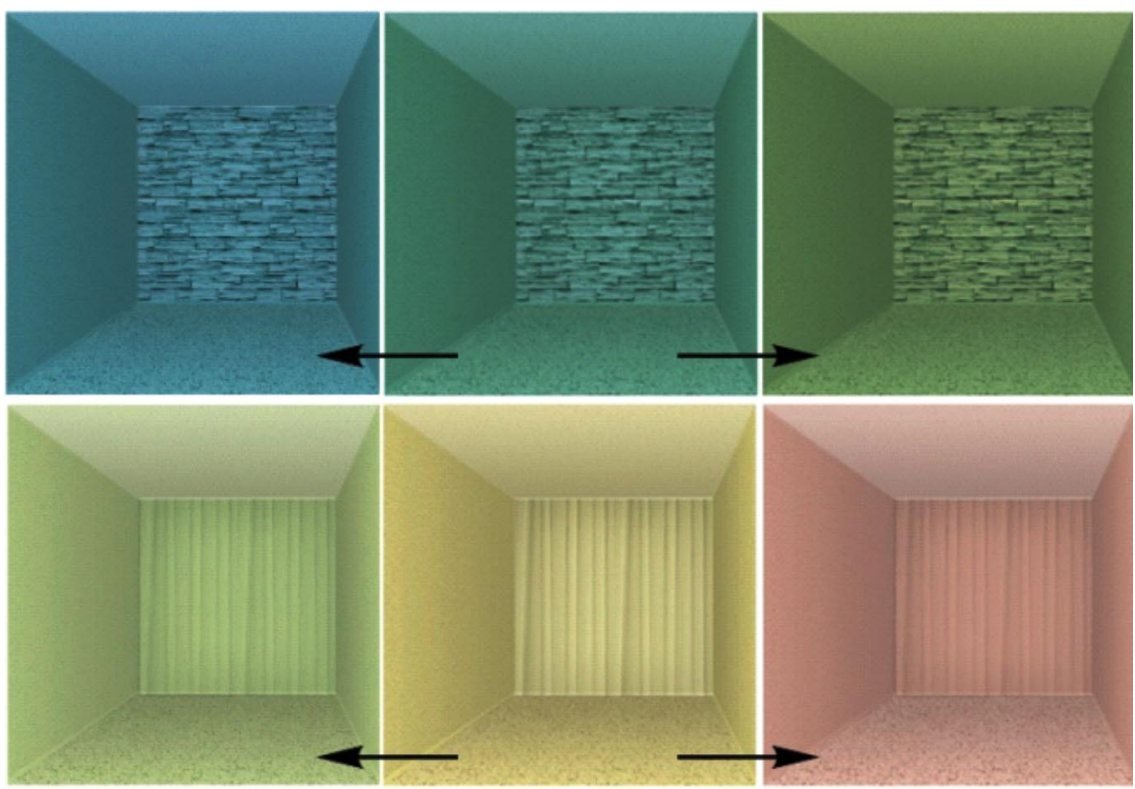

IF INPUT=9.5 
Figure 18: Spatial outputs generated for Size inputs
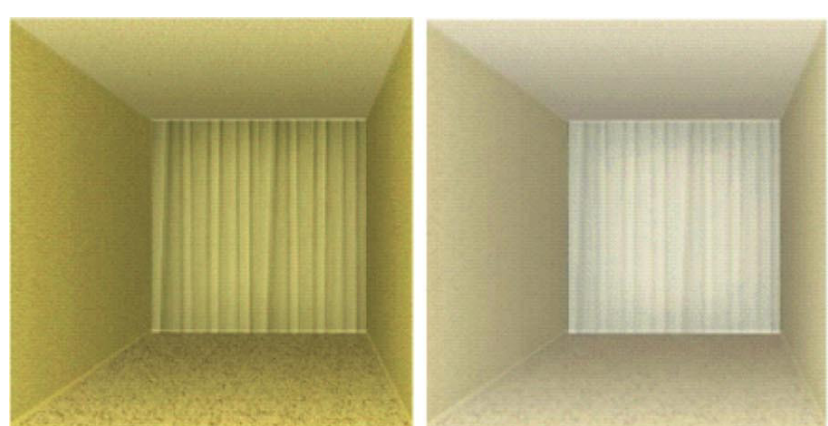

EXISTING IF INPUT $=3.5$
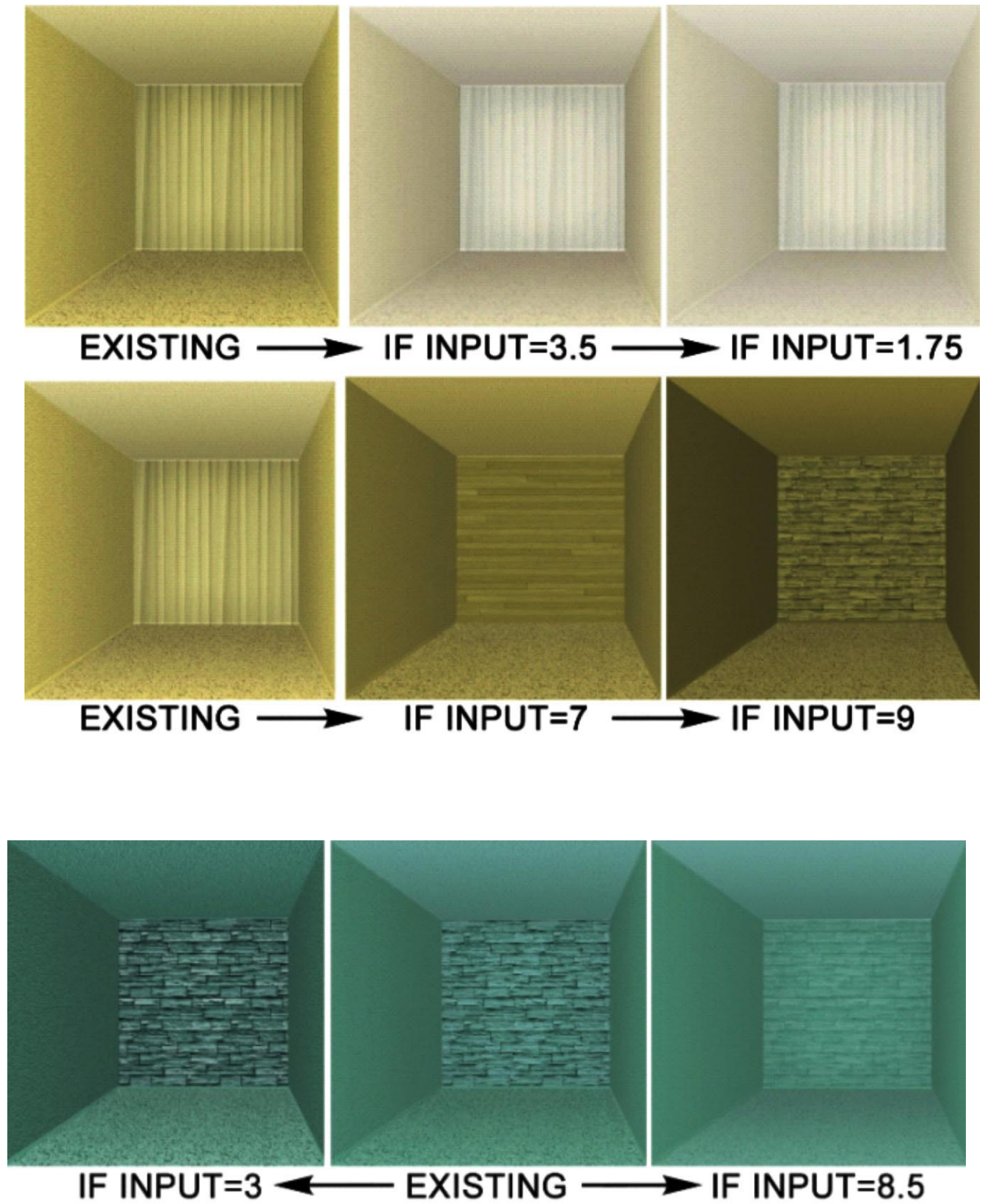

IF INPUT $=3 \longleftrightarrow$ EXISTING $\longrightarrow$ IF INPUT=8.5

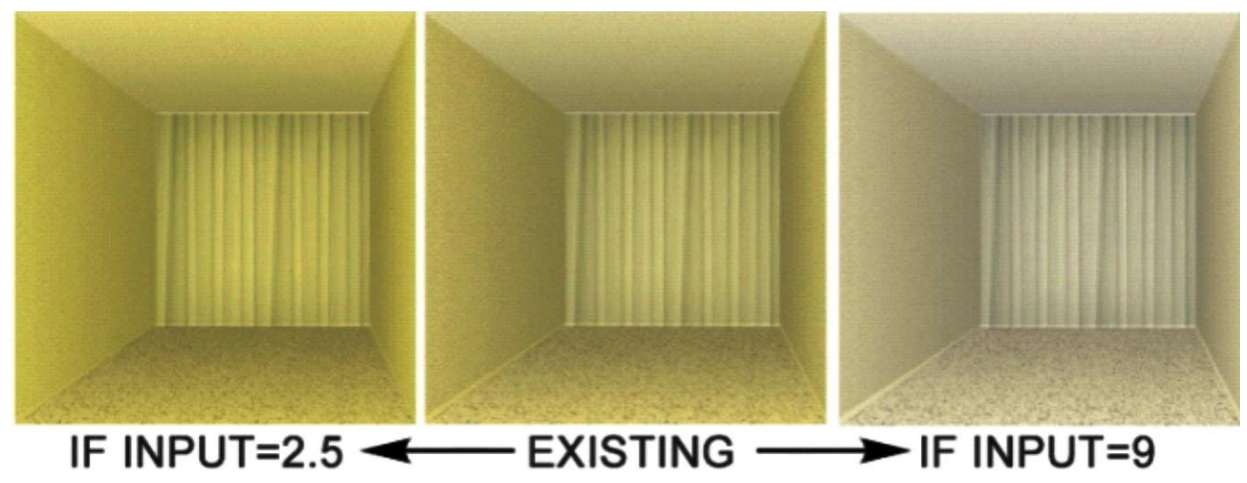

EXISTING
Figure 20: Spatial outputs generated for Softness inputs
Figure 19: Spatial outputs generated for Level of Arousal inputs 


\subsection{Inputs: Temperature}

Figure 17 illustrates temperature inputs and spatial outputs for both Space-A and Space-B. A high crisp input of 9.5 suggests that the user perceives the space as too warm. As an output, the system selects a surface hue that is cooler than the existing surface hue. On the other hand, a crisp input of 1 will generate a surface hue that is warmer.

\subsection{Inputs: Size}

Figure 18 illustrates size inputs and spatial outputs for Space-B. A lower size input of 3.5 suggests that the user perceives the space as quite small or intimate. The system will brighten surface color (increase value) to increase perceived spaciousness. An input of 1.75 will generate a surface color brighter than that. The fabric curtain wall, as the lightest material, will remain intact.

On the other hand, an input of 7 indicates that the user perceives the space as quite big or spacious. In order to make it feel more intimate, the system will darken surface color (decrease value) and change the fabric to wood, a heavier material. An input of 9 will generate an even darker surface color and a stone accent wall.

\subsection{Inputs: Level of Arousal}

The level of arousal inputs and spatial outputs for Space-A are illustrated in Figure 19. An input of 8.5 suggests that the user perceives the space as too exciting or stimulating. The system thus lowers the intensity of surface color (chroma) and smoothens the surface texture graniness to increase the feeling of calmness.

A lower input of 3, on the other hand, suggests that the user perceives the space as too calming. The system will increase the surface chroma and roughen the texture graininess to enhance the feeling of excitement.

\subsection{Inputs: Softness}

The user inputs of softness and corresponding spatial outputs are illustrated in Figure 20 for Space-B. A high crisp input of 9 suggests that the occupant perceives the space as too saturated or intense in color. The system will decrease the surface chroma to enhance the feeling of softness. A low crisp input of 2.5 will generate a surface color with high chroma.

\section{CONCLUSION}

The concept of space design proposed in this paper provides a basis for future research in the domain of intelligent spaces, i.e., their adaptive processes integrated with learning. The living space offers possibilities of spatial environments to the user through inferences drawn from user's subjective judgments. Interaction is based upon inferring meanings from perceptual data that may largely vary among a large population. As the design targets a large number of population, fuzzy logic approach was necessary to address the subjective uncertainties involved with sensory design parameters-color, texture, brightness and materials-that are non-quantifiable and perceptionbased. While certain objective affirmations could be confirmed (on color and brightness) in the Virtual Reality test, affective perception of sensory elements are considered highly subjective, as empirically found standardized rules are difficult to establish. Affective perceptions are also driven by identity, culture, values, mood or any other stimulus beyond the examined parameters. The mathematical model of fuzzy logic represents such ambiguity and unpredictability. It successfully allows the space to tailor itself to user goals and needs. 


\section{REFERENCES}

[1] Nadin M. Concepts and fuzzy logic. International Journal of General Systems 2012;41(8):860-7. Doi: 10.1080/ 03081079.2012 .726321

[2] Meech JA. How some simple fuzzy-thinking might have prevented the challenger accident. BISC discussion group; 7 April 2011.

[3] Naz A. Interactive living space design for neo-nomads: anticipation through spatial articulation. In: Nadin M, editor. Anticipation across disciplines, Heidelberg: Springer International Publishing; 2016. pp. 393-403.

[4] Ching FDK. Architecture: form, space, and order. Hoboken: Wiley; 2007.

[5] Franz G. An empirical approach to the experience of architectural space [dissertation]. Weimar: Max Planck Institute for Biological Cybernetics, Tübingen and the Bauhaus University; 2005.

[6] Naz A, Kopper R, McMahan RP, Nadin M. Emotional qualities of VR space. IEEE Virtual Reality Conference, Los Angeles, CA; 2017.pp. 3-11. doi:10.1109/VR.2017.7892225

[7] Naz A. Interactive living space for neo-nomads: an anticipatory approach [dissertation].Richardson: The University of Texas at Dallas; 2016.

[8] Riley NJ. S.F. supervisors back micro-apartments. San Francisco: SF Gate; [updated 2012 Nov 20; cited 2017 Sep 20] Available from:https://www.sfgate.com/bayarea/article/S-F-supervisors-back-micro-apartments-4055493.php

[9] Munsell.com [homepage on the Internet]. How color notations work. Munsell Color; [updated ; cited2016 Jan 30]Available from: http://munsell.com/about-munsell-color/how-color-notation-works/

[10] Zumthor, P. Thinking Architecture. Basel: Birkhäuser; 2010.

[11] Ponce-Cruz P, Ramirez-Figueroa FD. Intelligent control systems with LabVIEWTM. London: Springer; 2010.doi: 10.1007/ 978-1-84882-684-7.

[12] Ciftcioglu Ö. Design enhancement by fuzzy logic in architecture. In proceedings of the 12th IEEE International Conference on Fuzzy Systems (Vol. II), 25-28 May, 2003, St. Louis, Missouri: FUZZ-IEEE 2003: pp. 79-84.doi: 10.1109/ FUZZ.2003.1209341.

[13] Ciftcioglu Ö, Durmisevic, S. Fuzzy logic in architectural design. In proceedings of EUSFLAT 2001: the International Conference in Fuzzy Logic and Technology, 5-7 September, 2001, De Monfort University, Leicester, UK: pp. 265-268. 